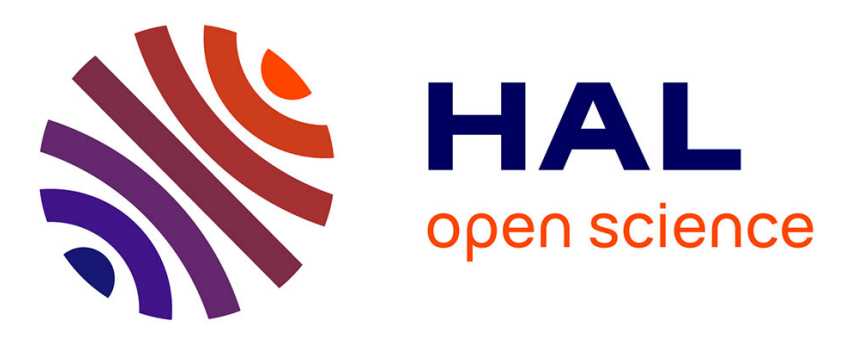

\title{
Improvement of the Magnetization of a Superconducting Bulk using an Iron Core
}

Bashar Gony, Kévin Berger, Bruno Douine, Michael Rudolf Koblischka, Jean

Lévêque

\section{- To cite this version:}

Bashar Gony, Kévin Berger, Bruno Douine, Michael Rudolf Koblischka, Jean Lévêque. Improvement of the Magnetization of a Superconducting Bulk using an Iron Core. IEEE Transactions on Applied Superconductivity, 2015, 25 (3), pp.8801005. 10.1109/TASC.2014.2373494 . hal-01077040

\section{HAL Id: hal-01077040 https://hal.science/hal-01077040}

Submitted on 23 Oct 2014

HAL is a multi-disciplinary open access archive for the deposit and dissemination of scientific research documents, whether they are published or not. The documents may come from teaching and research institutions in France or abroad, or from public or private research centers.
L'archive ouverte pluridisciplinaire HAL, est destinée au dépôt et à la diffusion de documents scientifiques de niveau recherche, publiés ou non, émanant des établissements d'enseignement et de recherche français ou étrangers, des laboratoires publics ou privés. 


\title{
Improvement of the Magnetization of a Superconducting Bulk using an Iron Core
}

\author{
B. Gony, K. Berger, B. Douine, M. R. Koblischka, and J. Lévêque
}

\begin{abstract}
In most studies, the magnetization was carried out without the iron core which is not the case in superconducting electrical applications. So in this paper we study the magnetization of YBCO bulk using a Pulsed Field Magnetization method in a symmetrical iron core. This iron core has the same structure as the iron core in an electrical motor. We have tested a new process of magnetization, firstly without the iron core and secondly with the iron core where we find an enhancement about $36 \%$ of the trapped magnetic field. The contribution of the iron core in this improvement was discussed. These experimentations help to carry out the magnetization of the superconducting bulk in the superconducting motor or in the coupling applications.
\end{abstract}

Index Terms - High-temperature superconductors, Magnetic cores, Magnetization, YBCO bulk.

\section{INTRODUCTION}

$\mathrm{T}$ HE new kinds of High Temperature Superconductors (HTS) are promising materials for the permanent magnet applications [1]-[5]. The superconducting bulks can play many roles in the industrial applications: the application of magnetic bearings [6], [7], and the application of high power density rotating machines [8]-[12] because it can trap or shield a high value of magnetic fields [13]-[16].

The maximum value of remnant magnetic induction in a permanent magnet is less than 1.5 Tesla for the neodymiumiron-boron to date. By using the principle of trapping the magnetic field in a high critical temperature superconductor, it is possible to obtain $4 \mathrm{~T}$ on the surface of a superconducting bulk at $77 \mathrm{Kelvin}$, i.e. in liquid nitrogen, and also up to $17.6 \mathrm{~T}$ at $26 \mathrm{~K}$ [14].

Furthermore, increasing the induction value is very important. For example in an application that aims to transfer an effort, by increasing the value of the induction produced, we can increase the value of the magnetic energy in the air gap and thus the electromagnetic torque.

There are several methods of magnetization but we chose the most convenient method to execute in situ, it is PFM for "Pulsed Field Magnetization" method. The general principle of this method is a discharge a capacitor bank in a coil which we

This work was supported in part by the ANR project REIMS, ANR-10BLAN-0944

B. Gony, K. Berger, B. Douine, and J. Lévêque, are with the University of Lorraine, GREEN, Research Group in Electrical engineering and Electronics of Nancy - EA 4366, Faculté des Sciences et Technologies - BP 70239, 54506 Vandoeuvre-lès-Nancy Cedex, France, e-mail: name.surname@univlorraine.fr.

M. R. Koblischka is with the Institute of Experimental Physics, Saarland University, Campus C63, 66123, Saarbrücken, Germany e-mail: m.koblischka@mx.uni-saarland.de. call the inductor. This discharge will generate a magnetic field pulse that will induce eddy currents and trapping a magnetic field in the superconductor bulk. This value of trapped magnetic field in the bulk depends on the critical current density $J_{c}$ of the bulk, the size of the bulk, and the form of the magnetic field pulse applied on the bulk [17]-[21].

In the literature, the study of the magnetization was carried out without an iron core [22]-[23], which is not the case in most of the electrical applications. In the traditional motors and in the magnetic coupling applications, an iron core exists, so it is really useful to study the magnetization of a superconducting bulk by using an iron core.

In our knowledge, any detailed study was never carried out concerning the magnetization by a PFM method in a symmetrical iron core. So, we tested and compared the magnetization by PFM method with/ without a symmetrical iron core. We also discussed the results and the role of the iron core in the magnetization process.

First, we present the experimental means that we use for magnetizing. Then we present the new applied process of magnetization that we have studied for two cases: without iron core and with an iron core. Finally, we compare the results and show the contribution of the iron core in the magnetization.

\section{EXPERIMENTAL MEANS}

In this section, we present the superconducting bulk, the inductor, the two principles of magnetization with and without iron core, and a new process of magnetization. The work was carried out at the temperature of liquid nitrogen $77 \mathrm{~K}$.

\section{A. Superconducting bulk}

We carried out all measurements on a superconducting bulk of YBCO which has a $31 \mathrm{~mm}$ of diameter, and $16.7 \mathrm{~mm}$ of height. The critical temperature of this superconducting bulk type is $92 \mathrm{~K}$. This bulk does not have any holes inside, Fig. 1.

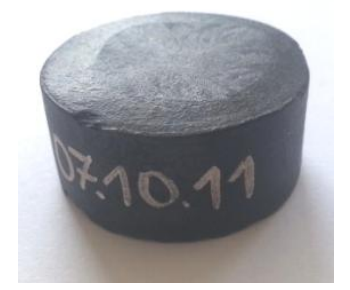

Fig. 1. The superconducting bulk used.

We always measure the magnetic field at the surface of superconducting bulk. We put two Hall sensors, HPP-NP from Arepoc, adapted to low temperatures: The first one is at the 
center of the bulk surface, and the second is on the edge of bulk surface, at $10 \mathrm{~mm}$ from the first sensor. The distance between the active part of the sensor and the surface of the bulk is $0.5 \mathrm{~mm}$.

\section{B. Inductor}

We use a copper coil as an inductor, Fig. 2, around the superconducting bulk due to its simple installation in industrial applications which has following parameters: 17 turns, an effective external diameter of $62 \mathrm{~mm}$, an effective internal diameter of $32.6 \mathrm{~mm}$, and an effective height of $20 \mathrm{~mm}$. The section of the copper is about $9 \mathrm{~mm}^{2}$ this section helps us to carry out some peaks of current with a current density more than $500 \mathrm{~A} / \mathrm{mm}^{2}$.

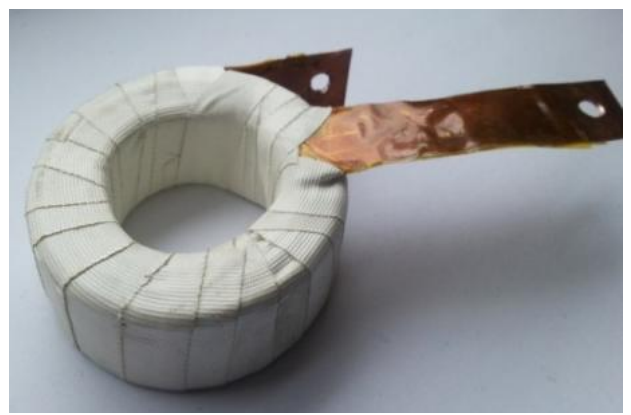

Fig. 2. The magnetization coil.

\section{Magnetization circuit and operating mode}

In order to apply the PFM method, we use an electrical circuit which consist of an autotransformer, a rectifier AC/DC, 24 capacitors in parallel for a total capacity of $79.2 \mathrm{mF}$, a thyristor, a diode, a coil, and some measuring devices, e.g. oscilloscope, voltmeter and amperemeter, see Fig. 3.

Basically, to carry out a magnetic field pulse, we should:

- charge the capacitor with a proper level of tension;

- discharge the capacitor in the copper coil.

We have done the magnetization in 2 different cases:

1. Without iron:

The first experimentation part is carried out without any iron in the vicinity of the superconductor, Fig. 4.

\section{With iron:}

The second one is carried out with an iron core which contains 200 sheets of isolated iron for decreasing the induced current in the magnetic core. Figure 5 shows a schematic of this E-I type iron core with the coil and the superconducting bulk inside it.

This magnetic circuit is almost the same as the one of a motor application like the synchronous motor proposed in
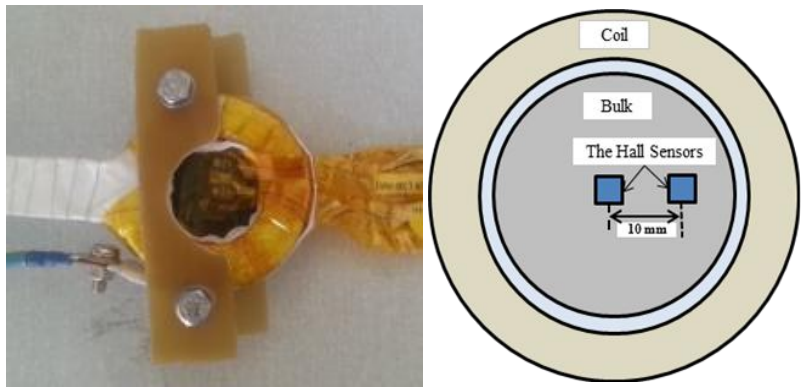

Fig. 4. The bulk YBCO inside its coil (left) and a sketch with the Hall sensors view (right).

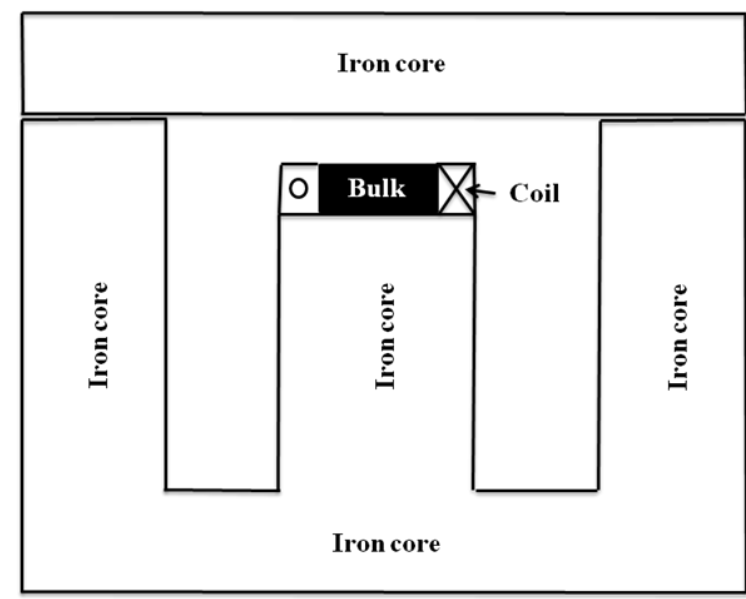

Fig. 5. A schematic of the E-I type iron core used.

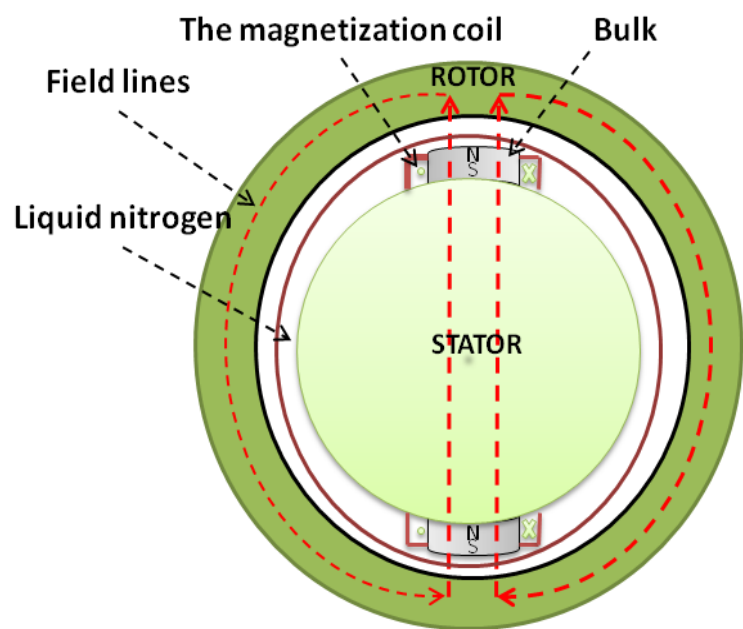

Fig. 6. A schematic of a motor with superconducting bulks.

Fig. 6 where the superconducting bulks are at the places of permanent magnets. Field lines will flow in the air, iron, and superconducting bulk.

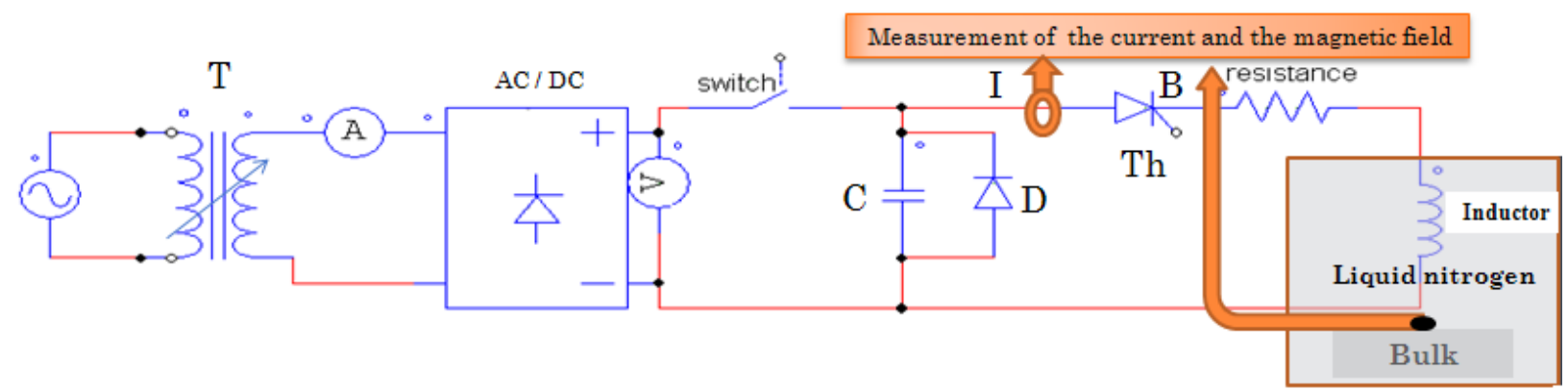

Fig. 3. The circuit of magnetization. 
Figure 7 shows the realized magnetic circuit that contains the magnetization coil, the superconducting bulk, and the two Hall sensors. The dimensions of this circuit magnetic are: $130 \mathrm{~mm}$ high, $150 \mathrm{~mm}$ of width and $74 \mathrm{~mm}$ depth. The air gap between the bulk and the iron core is about $7 \mathrm{~mm}$.

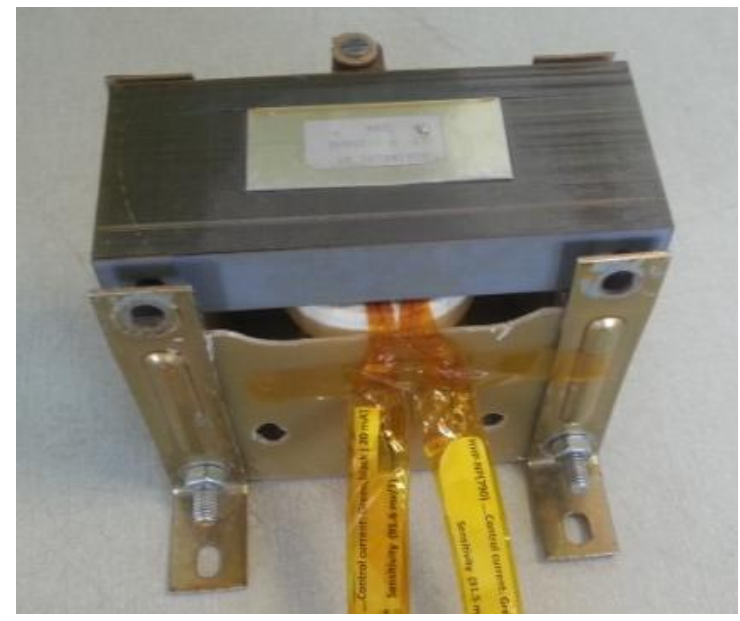

Fig. 7. The realized iron core for the experiments.

\section{PROCESS OF MAGNETIZATION}

This process is used for the two cases of magnetization, with and without iron. This process of magnetization is a new kind of direct magnetization, i.e. the bulk doesn't have any magnetic field when a higher magnetic field pulse is carried out. In the first part of this process, Fig. 8(a), one pulse is applied according to an initial charge voltage level of the capacitors and we save the maximum applied field and the trapped magnetic field in the bulk after 8-10 minutes. These values are saved for the two Hall sensors. Then we heat the bulk in order to remove the trapped magnetic field. Then we cool down the bulk in order to prepare it, and we apply a new magnetic pulse corresponding to an increase of $10 \mathrm{~V}$ applied to the capacitor bank. We repeat these steps: heating, cooling, applying a higher pulse, and we save the results until the maximum of trapped field is reached.

In the Figure $8(\mathrm{a})$, four increasing magnetic pulses are applied in phases $\mathrm{A}, \mathrm{B}, \mathrm{C}$, and D. $B_{t}^{\mathrm{A}}, B_{t}^{B}, B_{t}^{\mathrm{C}}$, and $B_{t}^{\mathrm{D}}$ are the stable trapped magnetic fields after 8-10 min at the end of the pulses. Where $B_{t}^{\mathrm{C}}>B_{t}^{\mathrm{A}}, B_{t}^{B}$, and $B_{t}^{\mathrm{D}}$, so the charge voltage of the capacitors in phase $C$ leads to the best magnetic pulse.

We repeat the pulse C, 3 to 6 times, for enhancing the trapped magnetic field without heating the bulk. As an example, in Fig. 8(b), $B_{t}^{\mathrm{C} 3}>B_{t}^{\mathrm{C} 4}, B_{t}^{\mathrm{C} 2}$, and $B_{t}^{\mathrm{C} 1}$, so the optimal pulse is $\mathrm{C} 3$ where we find the optimal trapped magnetic field $B_{t}^{\mathrm{C} 3}$.

\section{RESULTS AND DISCUSSION}

\section{A. Without iron}

The process of magnetization was applied in the superconducting bulk, and we found the optimal magnetic pulse by repeating the best pulse 4 times. The Figure 9 shows this last pulse: The current pulse has a maximum value of $4745 \mathrm{~A}$, and a duration of $6 \mathrm{~ms}$. The magnetic pulse has a maximum value of $1.60 \mathrm{~T}$ on the bulk surface center, and a $0.54 \mathrm{~T}$ of stable trapped magnetic field after $10 \mathrm{~min}$. The maximum value measured by the second sensor at the edge is $1.12 \mathrm{~T}$, and the stable trapped magnetic field is $0.39 \mathrm{~T}$. These curves were taken from the oscilloscope, and filtered after that using Matlab.

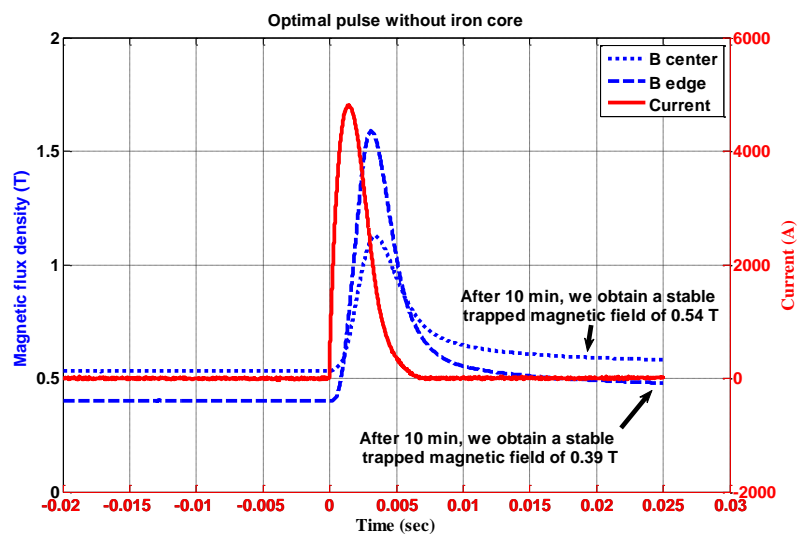

Fig. 9. Optimal magnetic and current pulse without any iron. Hall sensors are at the center and at the edge of the bulk.
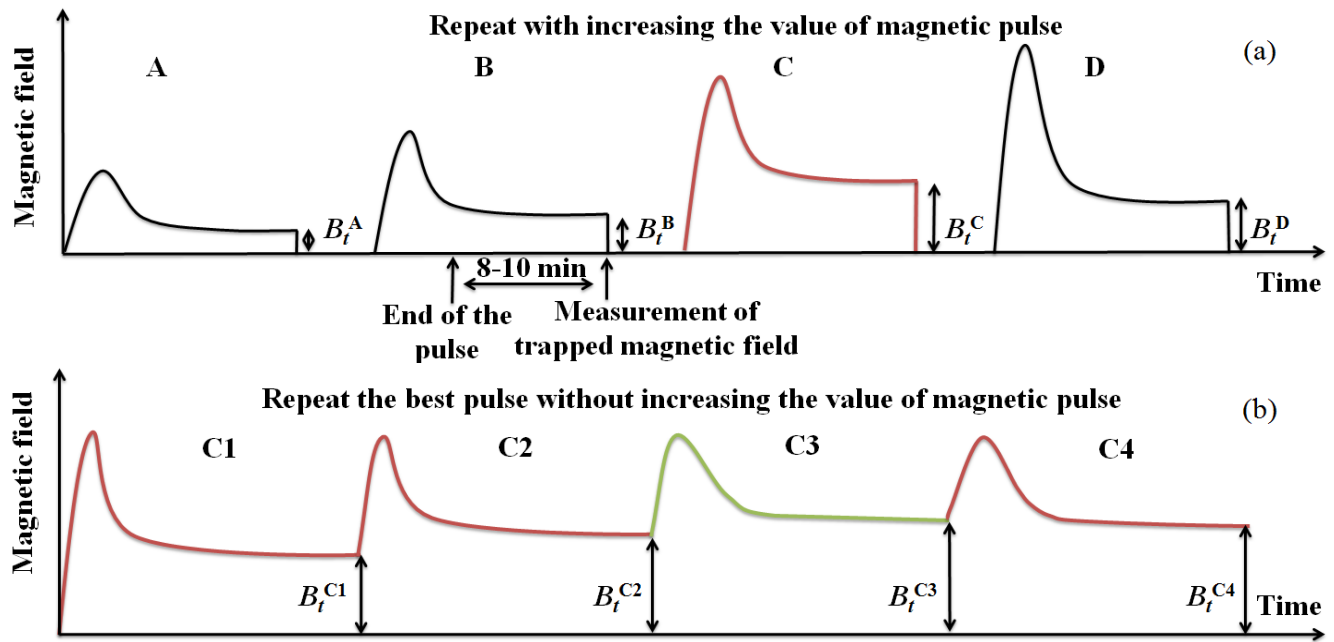

Fig. 8. (a) First part of the magnetization process. Finding the optimal charge voltage of the capacitors that leads to the best trapped magnetic field. (b) Second part of the magnetization process. Enhancement of the trapped magnetic field. 


\section{B. With iron}

By using the same process of magnetization, we find the optimal pulse by repeating the best pulse 5 times. The maximum value of the current is $2734 \mathrm{~A}$. The sensor at the center and the sensor at the edge measure a maximum value of magnetic flux density of $1.43 \mathrm{~T}$, and $1.79 \mathrm{~T}$ respectively. These sensors measure respectively a $0.85 \mathrm{~T}, 0.60 \mathrm{~T}$ of trapped magnetic flux density after 10 min, Fig. 10.

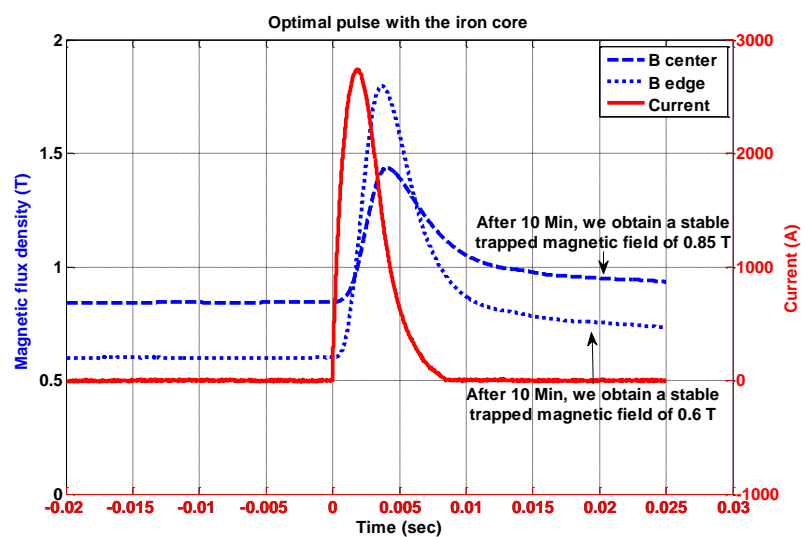

Fig. 10. Optimal magnetic and current pulse with the iron core. Hall sensors are at the center and at the edge of the bulk.

\section{Comparison with/without iron}

Figure 11 shows a magnetic field comparison between the magnetization with and without the iron core. We clearly see the enhanced trapped magnetic field with the iron core. We have an increasing of $36.5 \%$ of trapped magnetic field at the center of the bulk when we do the magnetization inside the iron core, and $35 \%$ at the edge of the bulk. In the case of the magnetization inside of the iron core, the maximum value of the current pulse for the optimal field is $43 \%$ lower of its value without the iron core. As we know that the magnetic field without the bulk reaction in the air gap is proportional to the value of the current pulse, so we also decrease the applied magnetic field by $43 \%$. We remark that the duration of the two current pulses is nearly the same.

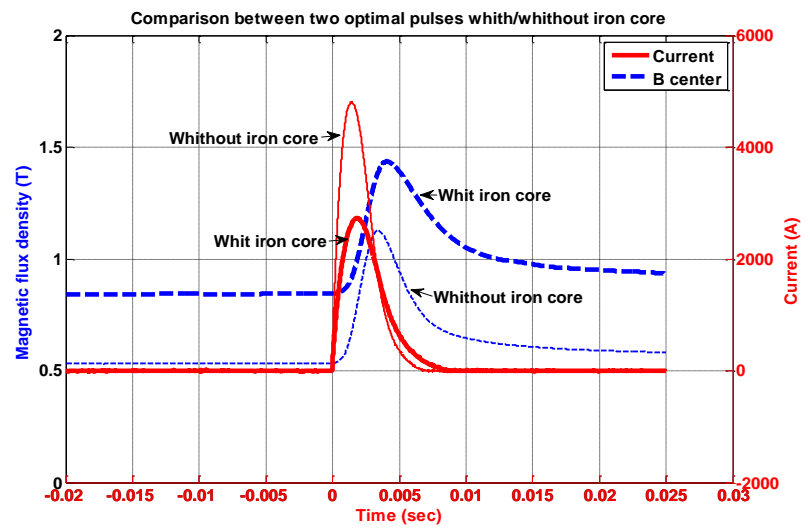

Fig. 11. Comparison the optimal magnetic pulses with and without iron core, and its currents.

In order to study the contribution of the iron core magnetization, we have done this test: we have removed the iron core after the stabilization, at point $\mathrm{A}$, and we observe the value of the trapped magnetic field, Fig. 12. After the new stabilization of the trapped magnetic field, we put again the superconducting bulk in the iron core, at point $\mathrm{B}$, where we find a new fast increase of the trapped magnetic field. After the new stabilization of the trapped field we remove the iron core a second time, at point $\mathrm{C}$, where we found also a fast decrease of the field. We put the iron core a second time, at point $\mathrm{D}$, and we can also observe a new increase of the trapped magnetic field in the same order of magnitude as for the previous case.

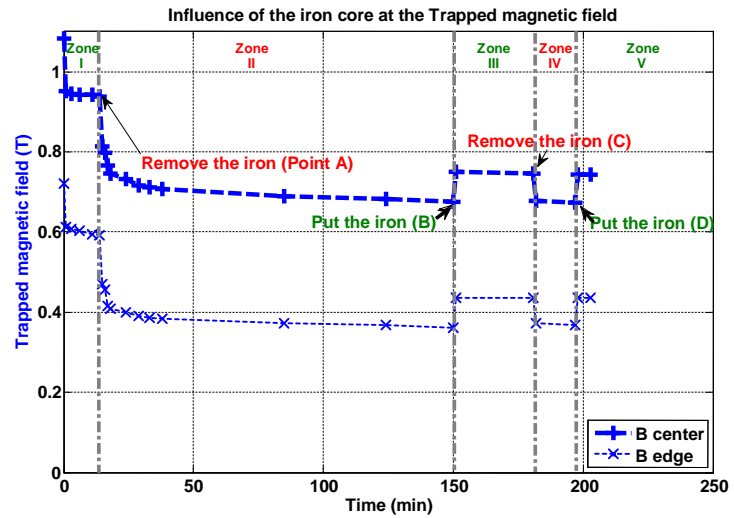

Fig. 12. The influence of the iron core on the trapped magnetic field in the superconducting bulk. The zones I, III, and V correspond to the evolution of the trapped magnetic field when the iron core is in place. Respectively, the zones II and IV correspond to the phases when the iron core is removed.

We can find that the decrease of the value of the trapped magnetic field between the level of the point $A$, and the level of the point $\mathrm{B}$ is due to the change of the magnetic field distribution applied on the superconducting bulk surface. This value is lost if we remove the iron, and we cannot reproduce it without a new magnetic pulse. When we put again the iron core in point $\mathrm{B}$, the fast increase of the magnetic field cannot be explained by current density redistribution in the superconductor. Moreover, the difference between the value at the points $\mathrm{B}$ and $\mathrm{C}$, is reproduced between the points $\mathrm{C}$ and $\mathrm{D}$. So we can conclude that this change of trapped magnetic field between the points $\mathrm{B}$ and $\mathrm{C}$, which is around $70 \mathrm{mT}$, is only due to the fact that a bigger part of the trapped magnetic flux is oriented in the direction of the iron core, the one that we measure. This phenomenon is reversible as for permanent magnets, and the stored energy in the YBCO bulk does not change.

As known, the trapped magnetic field can be increased by increasing the size of the YBCO bulk or by decreasing the cooling temperature. For the same size, at $30 \mathrm{~K}$ the trapped magnetic field can be more than 10 times the one at $77 \mathrm{~K}$ [13]. Some other experiments have to be done at lower temperature, in order to study the influence of the iron core on the magnetization process with higher trapped magnetic field.

\section{CONCLUSION}

Through our experimental results, we have proved than the magnetization of superconducting bulks using an iron core enhances the trapped magnetic field up to $36 \%$ compared to classical process without iron. A simple coil around the bulk is needed. With the use of iron, we have also decreased the current of the optimal pulse by $43 \%$. A new phenomenon concerning the iron core contribution have been clearly shown and explained. This study will help to carry out the magnetization of bulk superconductors for the superconducting motors or magnetic coupling applications. 


\section{REFERENCES}

[1] A. M. Campbell and D. A. Cardwell, "Bulk high temperature superconductors for magnet applications", Cryogenics, vol. 37, pp. 567-575, 1997.

[2] N. Yamachi, T. Nishikawa, M. Tomita, K. Sawa and M. Murakami, "Measurements of magnetic fields of levitated bulk superconductors", Physica C, vol. 378, pp. 877-882, 2002.

[3] M. Sander, "Cryo-permanent magnets-geometry, magnetization and cost issues", IEEE trans. Appl. Supercond., vol. 15, pp 1431-1434, 2005.

[4] T. H. Habisreuther, D. Litzkendorf, O. Surzehenko, M. Zeisberger, R. Muller, J. Riches, S. Scauroth, J. Dellith and W. Gawalek "Requirements on melt-textured Y-Ba-Cu-O for the use in magnetic bearings or electric motors" IEEE Trans. Appl. Supercond,. vol. 11, pp. 3501-3504, 2001.

[5] P. Vanderbemden, Z. Hong,T. A. Coombs, M. Ausloos, N. Hari Babu, D. A. Cardwell and A. M. Campbell, "Behavior of bulk high-temperature superconductors of finite thickness subjected to crossed magnetic fields: experiment and model", Supercond. Sci. Technol., vol. 17, 14 pages, 2007.

[6] T.A. Coombs, A. M. Campbell, R. storey and R. Weller, "Superconducting magnetic bearings for energy storage flywheels", IEEE trans. Appl. Supercond., vol. 9, pp. 968-971, 1999.

[7] T.M. Mulcahy, J.R. Hull, K. L. Uherkal, R. G. Abboud and J.J. Juna, "Test results of 2-kwh flywheel using passive pm and HTS bearings", IEEE Trans. Appl. Supercond., vol. 11, pp. 1729-1732, 2001.

[8] M. D. McCulloch and D. Dew-Hughes, "Brushless ac machines with high temperature superconducting rotors", Mater. Sci. Eng. B, vol. 53, pp.211-215, 1998.

[9] B. Oswald, K. J. Best, M. Setzer, M. Soll, W. Gawalek, A. Gutt, L. Kovalev, G. Krabbes, L. Fischer and H. C. Freyhardt, "Reluctance motors with bulk HTS material”, Supercond. Sci. Technol., vol. 18, s24, 2005.

[10] A. Alvarez, P. Suarez, D. Caceres, X. Granados, X. Obradors, R. Bosch, E. Cordero, B. Perez, A. Caballero and J. A. Blanco, "Superconducting armature for induction motor of axial flux based on YBCO bulks", Physica $C$, vol. 372-376, pp. 1517-1519, 2002.

[11] M. Qiu, H. K. Huo, Z. Xu, D. Xia, L. Z. Lin and G.M. Zhang "Technical analysis on the application of HTS bulk in permanent magnet motor", IEEE Trans. Appl. Supercond., vol. 15, pp. 3172-3175, 2005.

[12] G. Stumberger, M. T. Aydemirt, D. Zarko, T. A. Lipo, "Design of a linear bulk superconductor magnet synchronous motor for electromagnetic aircraft launch systems", IEEE trans. Appl. Supercond, vol. 14, pp. 54-62, 2004.

[13] M. Tomita and M Murakami "High-temperature superconductor bulk magnets that can trap magnetic fields of over 17 tesla at $29 \mathrm{~K}$ ", Nature, vol. 421, pp. 517-520, 2003.

[14] J. H. Durrell, A. R. Dennis, J. Jaroszynski, M. D. Ainslie, K. G. B. Palmer, Y-H. Shi, A. M. Campbell, J. Hull, M. Strasik, E. E. Hellstrom, D. A. Cardwell, "A Trapped Field of 17.6 T in Melt-Processed, Bulk Gd-Ba-Cu-O Reinforced with Shrink-Fit Steel”, Supercond. Sci. Technol., vol. 27, 082001, 2014.

[15] M. Eisterer, S. Haindl, M. Zehetmayer, R. Gonzalez-Arrabal, H. W. Weber, D. Litzkendorf, M. Zeisberger, T. Habisreuther, W. Gawalek, L. Shlyk and G. Krabbes "Limitations for the trapped filed in large grain YBCO superconductors", Supercond. Sci. Technol, vol. 19, s530, 2006.

[16] M. Muralidhar, M. R. Koblischka and M. Murakami "Improvement of critical current densities in bulk superconductors of the 123-Type", Physica stat. sol., vol. 171, pp. R7-R8, 1999.

[17] B. Douine, F. Sirois, J. Leveque, K. Berger, C.H. Bonnard, T. Hoang, and S. Mezani "A new direct magnetic method for determining in bulk superconductors from magnetic field diffusion measurements', IEEE trans. Appl. Supercond., vol. 22 (3), 9001604, 2012.

[18] G. P. Lousberg, M. Ausloos, P.H. Vanderbemden and B Vanderheyden "Bulk high-Tc superconductors with drilled holes: how to arrange the holes to maximize the trapped magnetic flux", Supercond. Sci. Technol., vol. 21 (2), $025010,2008$.

[19] L. Chen, Y.S. Cha, H. Claus, H. Zheng, B. W. Veal, and F. Z. Peng, "Optimization of pulsed-current profile for magnetizing high Tc bulk YBCO superconductors“, IEEE transactions on plasma science, vol. 34, pp. 1702$1708,2006$.

[20] K. Berger, J. Leveque, D. Netter, B. Douine, A. Rezzoug, "Influence of Temperature and/or Field Dependences of the E-J Power Law on Trapped Magnetic Field in Bulk YBaCuO”, IEEE trans. Appl. Supercond, vol. 17 (2), pp. 3028-3031, 2007.

[21] B. Gony, R. Linares, Q. Lin, K. Berger, B. Douine, and J. Leveque, "Influence of the inductor shape, and the magnetization processes on a trapped magnetic flux in a superconducting bulk", Physica C, vol. 503, pp. 1-6, 2014.
[22] U. Mizutani, T. Oka, Y. Itoh, Y. Yanagiy, M. Yoshikawa and H. Ikuta, "Pulsed-field magnetization applied to high-T $\mathrm{c}_{\mathrm{c}}$ superconductors", Appl. Supercond., vol. 6, pp. 235-246, 1998.

[23] L. Chen, Y. S. Cha, H. Claus H. Zheng, B.W. Veal and F.Z. Peng, "Optimization of pulsed-current profile for magnetizing high Tc bulk YBCO superconductors", IEEE Transactions on Plasma Science, Vol. 34, pp. 17021708, 2006. 